\title{
VOLATILE LOSS AND RETENTION ON KUIPER BELT OBJECTS
}

\author{
E. L. Schaller ${ }^{1}$ AND M. E. Brown ${ }^{1}$ \\ Received 2006 November 6; accepted 2007 February 23; published 2007 March 14
}

\begin{abstract}
Recent discoveries have shown that the very largest Kuiper Belt objects-Eris, 2005 FY9, and Sedna-are coated in methane and may contain other volatile ices as well. New detailed observations show that even within this class of volatile-rich bodies, unexpected differences exist in their surface compositions. 2005 FY9, a body approximately $60 \%$ the size of Pluto, with a reflectance spectrum similarly dominated by methane, has a surface depleted in molecular nitrogen by at least an order of magnitude with respect to Pluto. We find that the existence of this new class of volatile-rich objects, the lack of volatiles on most Kuiper Belt objects, and even the otherwise peculiar surface of 2005 FY9 can be explained as a consequence of atmospheric escape of volatile compounds. While previous studies of the surface compositions of objects in the Kuiper Belt have found no explainable patterns, atmospheric escape appears to provide a first-order explanation of the range of surface spectra seen on bodies in the outer solar system.
\end{abstract}

Subject headings: Kuiper Belt — planets and satellites: general

\section{INTRODUCTION}

Pluto and Neptune's satellite Triton have long been known to be covered in methane, molecular nitrogen, and carbon monoxide ices that sublime and form the tenuous atmospheres seen surrounding these bodies (Cruikshank et al. 1976, 1993; Cruikshank \& Silvaggio 1979; Owen et al. 1993). Until recently these volatile-rich surfaces appeared to be unique in the outer solar system. A decade of observations of small Kuiper Belt objects (KBOs) found that these bodies were covered in involatile water-ice or had flat spectra with nothing identifiable (Luu \& Jewitt 1998, 2001; Brown et al. 1999; Grundy et al. 2005; Trujillo et al. 2005). The recent discoveries of methane on large KBOs Eris, Sedna, and 2005 FY9 have shown that these objects are part of a new class of volatile-rich bodies in the outer solar system (Brown et al. 2005, 2007; Licandro et al. 2006a; Barucci et al. 2005). Even among these objects, however, there are differences in the relative abundances of the three volatile ices $\left(\mathrm{CH}_{4}, \mathrm{~N}_{2}\right.$, and $\left.\mathrm{CO}\right)$. For example, the spectrum of large KBO 2005 FY9 is dominated by broad absorption features due to long path lengths through solid $\mathrm{CH}_{4}$ with trace amounts of solid ethane also detected (Brown et al. 2007). The lack of detection of an $\mathrm{N}_{2}$ absorption feature, the long path lengths of $\mathrm{CH}_{4}$, and the detection of ethane were all interpreted as being due to a depletion of at least an order of magnitude of molecular nitrogen relative to methane on 2005 FY9 compared to Pluto (Brown et al. 2007).

To understand volatile loss and retention on KBOs, we have constructed a simple model of atmospheric escape that allows us to predict which bodies should be capable of retaining which surface volatile ices to the present day. While it has long been expected that large and cold bodies in the outer solar system should have the potential of retaining their volatile ices (Stevenson 1993), the recent proliferation of new discoveries in the Kuiper Belt provides us with a range of bodies with varying sizes and orbits on which to test predictions of volatile loss and retention.

\footnotetext{
${ }^{1}$ Division of Geological and Planetary Sciences, California Institute of Technology, Pasadena, CA 91125.
}

\section{ATMOSPHERIC ESCAPE}

The materials from which the bodies in the outer solar system accreted were rich in volatile ices; small primordial bodies such as comets contain several percent fractions of methane, nitrogen, and carbon monoxide (Krankowsky et al. 1986; Eberhardt et al. 1987; Jessberger et al. 1989). When these volatile ices are accessible to the surface, they will sublime and possibly form a vapor pressure controlled atmosphere. Depending on the mass and temperature of the body, atmospheric escape can occur through a variety of processes.

On Earth, light elements like hydrogen are lost through the thermal evaporation of particles in the high-velocity tail of the Maxwellian distribution through a process known as Jeans escape. On low-mass KBOs, the heavier volatile species $\left(\mathrm{CH}_{4}, \mathrm{~N}_{2}\right.$, and $\mathrm{CO}$ ) can be efficiently lost via this process. An even faster loss process that can occur on low-mass or high-temperature objects is hydrodynamic escape. This process occurs when the atmosphere flows away from the body at supersonic speeds such as occurs on comets. However, even on Pluto and Triton, where the thermal structures of the atmospheres are well known, it is difficult to calculate a priori from what regime (Jeans or hydrodyamic) escape is occurring. In addition, in cases of bodies with eccentric orbits like Pluto, the escape regime may vary over the course of the orbit (Trafton et al. 1997).

Of the two processes, Jeans loss provides a lower limit to the escape flux, but even with Jeans loss the escape rate is a very sensitive function of the temperature and height of the exobase, the location from which the escape is occurring. EUV heating of the upper atmosphere can cause the exobase temperature to be greatly elevated over the surface radiative equilibrium temperature, significantly increasing the escape rate. Consequently, the exobase temperature-and thus the true escape rate-cannot be calculated without a detailed knowledge of the thermal structure and composition of the atmosphere. While an accurate estimate of the true escape flux appears impossible, a reasonable lower bound to the escape rate can be found by simply assuming that the exobase is at the surface and by calculating the Jeans escape flux using the surface radiative equilibrium temperature. This calculated escape flux will be lower than any actual flux from any atmosphere where the exobase is elevated above the surface and has a temperature equal to or greater than the surface temperature. The only sit- 
uations in which this lower bound fails is for an atmosphere with an exobase temperature lower than the surface temperature. All comparable planetary atmospheres suffer EUV heating in the upper atmosphere and have elevated temperatures; thus, we deem this possibility remote and consider the surface Jeans escape rate to be a reasonable lower bound when the details of individual KBO atmospheres are unknown.

We model the Jeans escape flux (Chamberlain \& Hunten 1989) of $\mathrm{CH}_{4}, \mathrm{~N}_{2}$, and $\mathrm{CO}$ from a body with radius $R$ with a surface in radiative equilibrium as

$$
\frac{d M_{\mathrm{vol}}}{d t}=4 \pi R^{2} \frac{P_{\mathrm{vap}}(T)}{2 \sqrt{\pi} k T} \sqrt{\frac{2 k T}{m}}(1+\lambda) e^{-\lambda},
$$

where

$$
\lambda=\frac{G M m}{k T R},
$$

$d M_{\mathrm{vol}} / d t$ is the rate of loss of a given volatile in molecules per second, $P_{\text {vap }}(T)$ is the vapor pressure of a given volatile compound (Lodders \& Fegley 1998) at $T$, the radiative equilibrium temperature, $k$ is the Boltzmann constant, $m$ is the molecular mass of a given volatile species, $G$ is the gravitational constant, and $M$ is the mass of the body.

To explore volatile loss on bodies in the outer solar system, we calculated the minimum volatile loss a body of a given size and temperature could have experienced and compared it to the maximum initial volatile inventory it could have accessible to the surface. If a body is capable of losing its total inventory of volatiles through the slowest loss mechanism (Jeans escape assuming the surface radiative equilibrium temperature), examination of other loss processes (such as hydrodynamic escape, Jeans escape occurring from a higher temperature exobase, UV photolysis) is unnecessary as these processes will only deplete volatiles even faster. In addition, the mass of accessible volatile ices may be significantly less than the maximum initial inventory because volatiles may be locked away at depth and shielded from the surface, making depletion of surface volatiles occur even more rapidly. Therefore, for a body of a given size and surface temperature and assuming all volatiles are accessible to the surface, we calculate the total minimum volatile loss from Jeans escape over the age of the solar system.

In Figure 1 we show where this total minimum loss is equal to the approximate initial inventory of these volatiles for a given size and temperature assuming a density of $1.8 \mathrm{~g} \mathrm{~cm}^{-3}$. We assumed initial volatile inventories equal to the maximum gas production rate relative to water released from the nucleus of Halley's comet for $\mathrm{CH}_{4}, \mathrm{~N}_{2}$, and $\mathrm{CO}(0.07,0.02$, and 0.07 , respectively; Jessberger et al. 1989) and scaled by the rockwater mass fraction of Charon (Gulbis et al. 2006). Note that changing these initial volatile abundances by an order of magnitude does not appreciably change the positions of the lines in Figure 1 as they are controlled mainly by the vapor pressures of the compounds. Small and hot objects will have lost all of their accessible volatiles due to Jeans escape, while large and cold objects will not have necessarily been depleted in these volatiles via this process. Objects between the extremes are capable of losing some volatile species while retaining others.

In order to model minimum volatile loss on the currently known KBOs, we assume that all bodies began as volatile-rich, high-albedo objects similar to Pluto with all volatiles at the surface equilibrium temperature and accessible to the surface. For each of the known KBOs, we numerically calculate the volatile loss by dividing the object's orbit into small segments and integrating the total loss volatile over the age of the solar system. Finally, we translate this total mass loss into a volatile loss equivalent temperature corresponding to the temperature of an identical mass body in a circular orbit that would have experienced the same loss over the age of the solar system. The equivalent volatile loss temperature for most bodies is very close to the perihelion temperature since most of the loss occurs near perihelion. However, for objects with extremely eccentric orbits the equivalent temperature can be somewhat lower.

\section{RESULTS AND DISCUSSION}

The known KBOs divide into three general categories in this analysis (Fig. 1). (1) The large majority are too small and too hot to have retained any surface volatiles even with this minimum escape mechanism. (2) A small number of the largest objects have such low escape fluxes that even escape mechanisms many orders of magnitudes more effective will not be able to deplete their volatiles. (3) Three objects (2003 EL61, Quaoar, and 2005 FY9) exist near the transition between possible volatile retention and certain volatile loss.

The first two categories predicted by the atmospheric escape model perfectly reflect known KBO surface compositions. No KBOs that are predicted to be too small and hot to preserve volatiles have had volatiles detected on their surfaces, while all of the KBOs that are predicted to be large and cold enough to easily maintain volatiles indeed have had volatiles detected (Table 1). If volatiles were to be detected on the surface of any of the small hot bodies, their unexpected presence would have

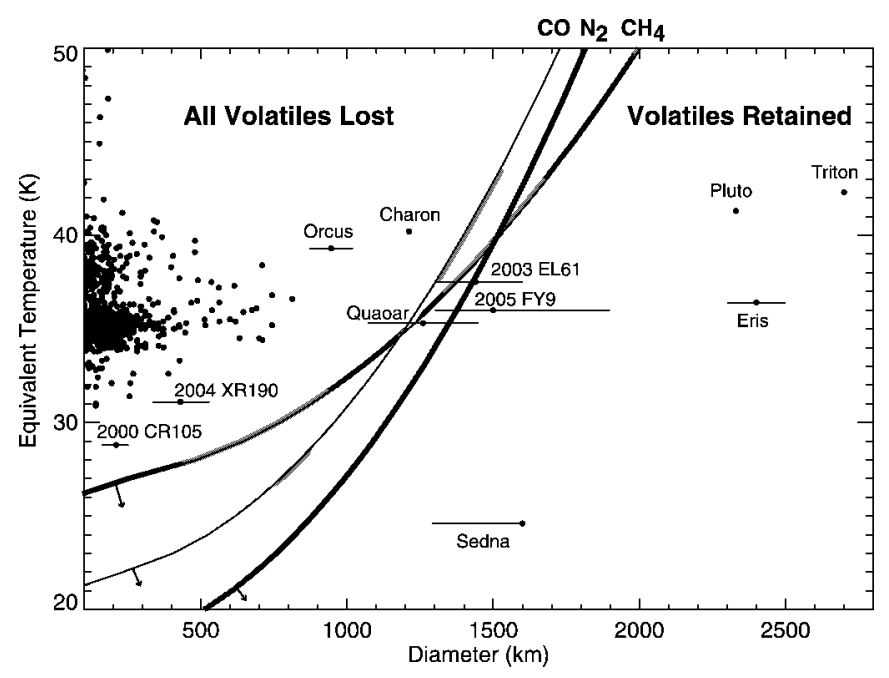

FIG. 1.-Minimum volatile loss in the outer solar system as a function of temperature and radius. The lines show the temperatures as a function of radius at which the initial inventories of $\mathrm{CH}_{4}, \mathrm{~N}_{2}$, and $\mathrm{CO}$ must be lost over the age of the solar system. Note that changing these initial volatile abundances by an order of magnitude does not appreciably change the positions of the lines as they are controlled mainly by the vapor pressures of the compounds. The arrows show the distance each line would congruently shift if we assumed only $10 \%$ of the initial volatile inventory is accessible to the surface. The known KBOs are plotted on this figure by considering their equivalent volatile loss temperatures. To find this temperature we calculate the loss of $\mathrm{N}_{2}, \mathrm{CH}_{4}$, and $\mathrm{CO}$ at each position in the object's orbit and integrate the total loss over the age of the solar system. We then translate this total mass loss into a temperature corresponding to the temperature of an identical body in a circular orbit that would have experienced the same loss over the age of the solar system. For KBOs with unknown sizes, we assumed an albedo of 0.1 to calculate the size. 
TABLE 1

Sizes, Orbits, and Surface Characteristics of KBOs Labeled on Figure i

\begin{tabular}{|c|c|c|c|c|c|c|}
\hline Body & $\begin{array}{c}\text { Diameter } \\
(\mathrm{km})\end{array}$ & $\begin{array}{c}\text { Col. (2) } \\
\text { References }\end{array}$ & $\begin{array}{l}\text { Perihelion } \\
\text { (AU) }\end{array}$ & $\begin{array}{l}\text { Aphelion } \\
\text { (AU) }\end{array}$ & Known Surface Volatiles & $\begin{array}{c}\text { Col. (6) } \\
\text { References }\end{array}$ \\
\hline Eris ......... & $2400 \pm 100$ & 1 & 37.8 & 97.6 & $\mathrm{CH}_{4}, \mathrm{~N}_{2}$ & 2,3 \\
\hline Pluto .......... & $2328 \pm 46$ & 4 & 29.7 & 49.3 & $\mathrm{CH}_{4}, \mathrm{~N}_{2}, \mathrm{CO}$ & 5,6 \\
\hline Charon...$\ldots \ldots \ldots$ & $1212 \pm 16$ & 7 & 29.7 & 49.3 & None & 8 \\
\hline Triton ... & 2700 & 9 & 29.8 & 30.3 & $\mathrm{CH}_{4}, \mathrm{~N}_{2}, \mathrm{CO}$ & 10,11 \\
\hline 2003 EL61 ......... & $2000 \times 1500 \times 1000$ & 12 & 35.1 & 51.5 & None & 13 \\
\hline Sedna & $<1600$ & 14 & 76.2 & 975.1 & $\mathrm{CH}_{4}, \mathrm{~N}_{2}$ ? & 15 \\
\hline 2005 FY9 & $1500 \pm 300$ & 14 & 38.5 & 53.1 & $\mathrm{CH}_{4}$ & 16,17 \\
\hline Quaoar ........... & $1260 \pm 190$ & 18 & 41.9 & 44.9 & None, $\mathrm{CH}_{4}$ ? & 19,20 \\
\hline Orcus $\ldots \ldots \ldots \ldots$ & $950 \pm 70$ & 14 & 30.5 & 48.3 & None & 21,22 \\
\hline 2004 XR190 ....... & $335-530^{\mathrm{a}}$ & $\ldots$ & 52.3 & 61.8 & Unknown & $\ldots$ \\
\hline 2000 CR105 ....... & $160-253^{\mathrm{a}}$ & $\ldots$ & 44.4 & 397.6 & Unknown & $\ldots$ \\
\hline
\end{tabular}

${ }^{a}$ Diameter range estimated by assuming $10 \%-25 \%$ albedo.

RefERENCES. - (1) Brown et al. 2006a; (2) Brown et al. 2005; (3) Licandro et al. 2006b; (4) Young \& Binzel 1993; (5) Cruikshank et al. 1976; (6) Owen et al. 1993; (7) Gulbis et al. 2006; (8) Brown \& Calvin 2000; (9) Smith et al. 1989; (10) Cruikshank \& Silvaggio 1979; (11) Cruikshank et al. 1993; (12) Rabinowitz et al. 2006; (13) Trujillo et al. 2007; (14) Stansberry et al. 2007; (15) Barucci et al. 2005; (16) Licandro et al. 2006a; (17) Brown et al. 2007; (18) Brown \& Trujillo 2004; (19) Jewitt \& Luu 2004; (20) Brown 2003; (21) de Bergh et al. 2005; (22) Trujillo et al. 2005.

to be explained by a recent impact or other transient event that liberated stored volatiles from the subsurface or by a geochemical mechanism such as serpentinization that produced volatiles in the interior of the body.

The most interesting behavior occurs for the three objects near the line between certain loss and possible volatile retention. The transition region is also the location where different volatiles will be lost at different rates. For the lower mass or higher temperature bodies, vapor pressure dominates over gravitational attraction, so the volatile species with higher vapor pressures, like $\mathrm{N}_{2}$ and $\mathrm{CO}$, will escape the fastest. On the higher mass or lower temperature bodies, in contrast, gravitational attraction is dominant and lower molecular weight species, like methane, will escape the fastest.

The first transition object, 2003 EL61, is one of the most unusual bodies in the solar system. This rapidly spinning $2000 \times 1500 \times 1000 \mathrm{~km}$ body (Rabinowitz et al. 2006) has a spectrum dominated by water ice and has no evidence for the presence of any volatile ices (Trujillo et al. 2007). However, the high density, fast rotation rate, and multiple satellite system of 2003 EL61 (Brown et al. 2006b) suggest that it experienced a disruptive collision where it lost most of its water ice and likely all of its volatiles as well.

Quaoar is also in the transition region between complete volatile loss and possible volatile retention. This object has a water-ice-dominated spectrum with an additional absorption feature at $2.2 \mu \mathrm{m}$ that has not been seen on any other smaller KBOs. The feature has been interpreted as an absorption due to nonvolatile ammonia ices (Jewitt \& Luu 2004), but it has also been argued to be due to trace amounts of methane on the surface (Brown 2003), consistent with Quaoar being an object that may still retain some initial volatiles. Higher quality spectra to explore the surface of this object are clearly warranted.
The final transition object is the methane-rich but nitrogendepleted 2005 FY9. 2005 FY9 exists near the region where $\mathrm{N}_{2}$ must be depleted but $\mathrm{CH}_{4}$ and $\mathrm{CO}$ can possibly remain. The formerly unexplained depletion of nitrogen seen in the spectrum of this object may be explained by differential atmospheric loss of $\mathrm{N}_{2}$ relative to $\mathrm{CH}_{4}$. The presence of volatiles on 2005 FY9 appears to rule out hydrodyamic escape occurring over the age of the solar system on this body. The minimum hydrodynamic escape rate is nearly 2 orders of magnitude greater than the Jeans escape rate for a body of this size and temperature.

Atmospheric loss appears to explain not only the presence and absence of volatiles, but may also be able to explain the balance of the individual volatile ices present bodies of the outer solar system. More detailed modeling would be required to predict more accurate escape rates, but such modeling does not appear necessary for an initial description of the range of spectra presently seen on bodies in the outer solar system. While it is perhaps surprising that the simple lower bound escape rate seems to accurately predict loss on the transition objects, it may be that initial loss rates are higher but that this mechanism is the rate limiting step when objects are close to depleting all of their volatiles. Objects could thus spend significant time periods near this transition region. As more objects are discovered in the Kuiper Belt with high perihelia orbits similar to Sedna's, we expect that all but the smallest of these objects could contain volatile ices on their surfaces and may show depletions in nitrogen similar to 2005 FY9.

We thank John Stansberry for a helpful review and David Stevenson, Kristina Barkume, Henry Roe, Oded Aharonson, and Re'em Sari for helpful discussions.

\section{REFERENCES}

Barucci, M. A., Cruikshank, D. P., Dotto, E., Merlin, F., Poulet, F., Dalle Ore, C., Fornasier, S., \& de Bergh, C. 2005, A\&A, 439, L1

Brown, M. E. 2003, AAS DPS Meeting, 35, 29.01

Brown, M. E., Barkume, K. M., Blake, G. A., Schaller, E. L., Rabinowitz,

D. L., Roe, H. G., \& Trujillo, C. A. 2007, AJ, 133, 284

Brown, M. E., \& Calvin, W. M. 2000, Science, 287, 107

Brown, M. E., Schaller, E. L., Roe, H. G., Rabinowitz, D. L., \& Trujillo,

C. A. 2006a, ApJ, 643, L61

Brown, M. E., \& Trujillo, C. A. 2004, AJ, 127, 2413
Brown, M. E., Trujillo, C. A., \& Rabinowitz, D. L. 2005, ApJ, 635, L97 Brown, M. E., et al. 2006b, ApJ, 639, L43

Brown, R. H., Cruikshank, D. P., \& Pendleton, Y. 1999, ApJ, 519, L101

Chamberlain, J. W., \& Hunten, D. M. 1989, Theory of Planetary Atmospheres (2nd ed.; San Diego: Academic Press)

Cruikshank, D. P., Pilcher, C. B., \& Morrison, D. 1976, Science, 194, 835

Cruikshank, D. P., Roush, T. L., Owen, T. C., Geballe, T. R., de Bergh, C.,

Schmitt, B., Brown, R. H., \& Bartholomew, M. J. 1993, Science, 261, 742 Cruikshank, D. P., \& Silvaggio, P. M. 1979, ApJ, 233, 1016 
de Bergh, C., Delsanti, A., Tozzi, G. P., Dotto, E., Doressoundiram, A., \& Barucci, M. A. 2005, A\&A, 437, 1115

Eberhardt, P., et al. 1987, A\&A, 187, 481

Grundy, W. M., Buie, M. W., \& Spencer, J. R. 2005, AJ, 130, 1299

Gulbis, A. A. S., et al. 2006, Nature, 439, 48

Jessberger, E. K., Kissel, J., \& Rahe, J. 1989, in Origin and Evolution of Planetary and Satellite Atmospheres, ed. S. K. Atreya, J. B. Pollack, \& M. S. Matthews (Tuscon: Univ. Arizona Press), 167

Jewitt, D. C., \& Luu, J. X. 2001, AJ, 122, 2099 2004, Nature, 432, 731

Krankowsky, D., et al. 1986, Nature, 321, 326

Licandro, J., Grundy, W. M., Pinilla-Alonso, N., \& Leisy, P. 2006a, A\&A, 458, L5

Licandro, J., Pinilla-Alonso, N., Pedani, M., Oliva, E., Tozzi, G. P., \& Grundy, W. M. 2006b, A\&A, 445, L35

Lodders, K., \& Fegley, B. 1998, The Planetary Scientist's Companion (New York: Oxford Univ. Press)
Luu, J. X., \& Jewitt, D. C. 1998, ApJ, 494, L117

Owen, T. C., et al. 1993, Science, 261, 745

Rabinowitz, D. L., Barkume, K., Brown, M. E., Roe, H., Schwartz, M., Tourtellotte, S., \& Trujillo, C. 2006, ApJ, 639, 1238

Smith, B. A., et al. 1989, Science, 246, 1422

Stansberry, J. A., et al. 2007, in Kuiper Belt, ed. M. A. Barucci et al. (Tucson: Univ. Arizona Press), in press

Stevenson, D. J. 1993, Lunar Planet. Sci. Conf., 24, 1355

Trafton, L. M., Hunten, D. M., Zahnle, K. J., \& McNutt, R. L., Jr. 1997, in Pluto and Charon, ed. S. A. Stern \& D. J. Tholen (Tuscon: Univ. Arizona Press) 475

Trujillo, C. A., Brown, M. E., Barkume, K. M., Schaller, E. L., \& Rabinowitz, D. L. 2007, ApJ, 655, 1172

Trujillo, C. A., Brown, M. E., Rabinowitz, D. L., \& Geballe, T. R. 2005, ApJ, 627, 1057

Young, E. F., \& Binzel, R. P. 1993, Icarus, 102, 134 